\title{
COMPULSÃO ALIMENTAR ANTES E APÓS A CIRURGIA BARIÁTRICA
}

\author{
Binge eating before and after bariatric surgery \\ Cristiane Evangelista MACHADO, Bruno ZILBERSTEIN, \\ Ivan CECCONELLO, Marlene MONTEIRO
}

ABCDDV/622

Machado CE, Zilberstins B, Cecconello I, Monteiro M. Compulsão alimentar antes e após a cirurgia bariátrica. ABCD Arq Bras Cir Dig 2008;21(4):185-91

RESUMO - Racional - A obesidade é distúrbio de origem multifatorial complexa que congrega aspectos de ordem psicológica, social, biológica e requer abordagem multidisciplinar. É considerada fator de risco para a compulsão alimentar, de modo que a dinâmica emocional do obeso contribuiria para desencadear o ato compulsivo. Objetivo - Analisar os indícios de compulsão alimentar em pacientes submetidos à cirurgia bariátrica, antes e dois anos após a operação. Métodos - Foi realizado estudo prospectivo em 50 pacientes submetidos à cirurgia bariátrica pela técnica de Fobi-Capella. Os instrumentos de avaliação psicológica, aplicados antes e após dois anos, foram o teste das Pirâmides Coloridas de Max Pfister e entrevista clínica semi-estruturada. O teste foi aplicado antes e após a operação para identificar indícios de compulsão. A entrevista somente no pósoperatório. Resultados - Citando somente os dados principais, os dados mostraram estabilidade e capacidade para lidar com conflitos em $16 \%$ dos pacientes antes da operação e em $10 \%$ após. Para a capacidade de controle e adaptação, $36 \%$ dos pacientes apresentavam fortes indícios de atitude de controle e rigidez antes da operação e $32 \%$ após. Quanto aos indicadores de impulsividade, agressividade e irritabilidade, houve fortes indícios de atitudes agressivas e impulsivas em $28 \%$ antes da operação e em $36 \%$ após. Quanto ao comportamento beliscador, eram presentes em $94 \%$ dos pacientes no pré-operatório e em $60 \%$ no pós. Quanto à preferência alimentar, foi a por alimentos calóricos sem menção à bebidas alcoólicas no pré-operatório, e no pós-operatório por alimentos de fácil ingestão. Quanto ao motivo atribuído aos episódios compulsivos, antes da operação $4 \%$ dos pacientes relataram estado de nervosismo, $24 \%$ afirmaram distúrbios de humor e $52 \%$ identificaram sentimentos de impotência ou submissão. No pós, foram $10 \%, 28 \%$ e $38 \%$. Quanto às respostas compensatórias, eram elas ingestão de doces em $12 \%$; ingestão de petiscos em $2 \%$ e comer indiscriminadamente tudo que estivesse ao alcance em $64 \%$ dos pacientes. Após, $18 \%, 10 \%$ e $22 \%$. Conclusão - Os pacientes apresentam indícios de compulsão alimentar antes e após a operação. Ela está relacionada a aspectos psicológicos. Os hábitos e preferências alimentares modificam-se após a operação, de modo que os pacientes passam a consumir alimentos de fácil ingestão frente a situações que os faziam comer compulsivamente, não sendo observado trocas de compulsão. O comportamento alimentar inadequado persiste ou é agravado após a operação.

DESCRITORES - Obsessão. Obesidade. Cirurgia bariátrica.

\section{INTRODUÇÃO}

A obesidade é distúrbio de origem multifatorial complexa que congrega aspectos de ordem psicológica, social, biológica e requer abordagem multidisciplinar para compreensão, diagnóstico e tratamento adequados. É considerada fator de risco para a compulsão alimentar, de modo que a dinâmica emocional do obeso contribuiria para desencadear o ato compulsivo?.

Diversos estudos demonstram a associação entre obesidade e compulsão alimentar, apresentando alta incidência na população que busca tratamento para redução de peso ${ }^{6,7,13,18,19,24}$. Grande parte destes pacientes apresenta episódios recorrentes de comer compulsivo, sem os comportamentos compensatórios característicos da bulimia nervosa. $\mathrm{O}$ besidade e compulsão alimentar podem ter relação causal na qual o comportamento compulsivo é o

Trabalho realizado Disciplina de Cirurgia do Aparelho Digestivo do Departamento de Gastroenterologia da Faculdade de Medicina da Universidade de São Paulo, São Paulo, SP, Brasil

Correspondência: crismachado07@yahoo.com.br agente causador ou facilitador da obesidade, assim como a gravidade da compulsão também parece estar relacionada com o grau de obesidade.

Estudos epidemiológicos demonstram compulsão alimentar em $15 \%$ a $50 \%$ dos pacientes que buscam tratamento para emagrecer, com tendência 1,5 maior para as mulheres ${ }^{1}$. No Brasil entre os portadores de obesidade que realizam a cirurgia bariátrica a prevalência é de $27 \%$ a $47 \%$, porém esta dimensão pode variar, em parte, devido às dificuldades diagnósticas ${ }^{1,22}$.

Os pacientes que apresentam compulsão alimentar têm maior frequência de recaídas após o tratamento para a perda de peso, maior comorbidades como depressão, abuso de álcool e drogas, transtornos de personalidade e insatisfação com a imagem corporal quando comparados com obesos-controle.

Na cirurgia bariátrica, em que existe redução do estômago, cria-se um componente restritivo no qual a quantidade de alimentos ingerida é intensamente diminuída, levando a perda de peso duradoura. A limitação imposta pela operação pode representar grande risco aos pacientes compulsivos, tornando-se difícil a adaptação à nova 
condição alimentar. Estudo com pacientes submetidos à cirurgia restritiva acompanhados por 10 anos, encontrando episódios bulímicos em $52 \%$ dos casos, transtorno do comer compulsivo em $16 \%$ e síndrome do comer noturno em $10 \%{ }^{5}$.

O impacto da compulsão alimentar pré-operatória nos resultados da cirurgia é pouco estudado ${ }^{5,12}$. Pacientes submetidos à cirurgia bariátrica necessitam compreender a dinâmica associada ao comportamento alimentar e desenvolver novos hábitos, para evitar que outros transtornos ocorram em compensação ao distúrbio alimentar. Desta forma, optou-se por estudar a compulsão alimentar após cirurgia bariátrica, através dos métodos proporcionados pela avaliação psicológica.

Assim, este trabalho tem por objetivos analisar os indícios de compulsão alimentar em pacientes submetidos à cirurgia bariátrica, pela técnica de Fobi-Capella, antes e pelo menos dois anos após a operação.

\section{MÉTODO}

Foi realizado estudo prospectivo em 50 pacientes submetidos à cirurgia bariátrica pela técnica de Fobi-Capella do Serviço de Cirurgia do Estômago da Divisão de Clínica Cirúrgica II do Hospital das Clínicas da Faculdade de Medicina da Universidade de São Paulo. Destes 43 (86\%) eram mulheres e sete (14\%) homens, com idade média de 42,4 anos (mínima 22 anos e máxima 65 anos) e IMC inicial médio de 48,5 (mínimo 37 e máximo 73), com tempo médio de seguimento pós-operatório de 36,6 meses (mínimo dois anos e máximo cinco anos).

Utilizou-se termo de consentimento livre e esclarecido para que os pacientes, depois de informados sobre os objetivos do estudo, autorizassem a participação na pesquisa.

\section{Instrumentos de avaliação psicológica}

Os pacientes foram submetidos à avaliação psicológica antes e dois a cinco anos após a operação. Os instrumentos utilizados foram o teste das Pirâmides Coloridas de Max Pfister e entrevista clínica semi-estruturada. O teste foi aplicado antes e após a operação para identificar indícios de compulsão. A entrevista, somente no pós-operatório possibilitando o relato dos pacientes sobre hábitos alimentares e mecanismos associados à compulsão antes e após a operação.

\section{Teste das Pirâmides Coloridas de Max Pfister}

Este instrumento utiliza o estudo da forma e cores de pirâmides para identificar aspectos de personalidade. A avaliação é baseada na relação entre cor e estados emocionais. É um instrumento de diagnóstico da dinâmica emocional e de habilidades cognitivas. Compõe-se de três cartelas contendo um esquema de pirâmide, folha de avaliação e mostruário de quadrículos contendo 10 cores subdivididas em diferentes tonalidades, variando de tons claros a escuros. Cada pirâmide possui 15 espaços a serem preenchidos com os quadrículos coloridos. Solicita-se a construção de três pirâmides, utilizando as cores que o indivíduo considere bonitas. Procede-se a anotação das formas e das cores utilizadas.

Finalizada a aplicação, é realizada a contagem das cores de cada pirâmide para fazer a análise do resultado. A pontuação de cada cor é transformada em porcentagem para que se possa verificar a incidência das cores e classificá-las em baixa (-1), média (0) e alta (1) incidência. As variáveis indicadas por cada cor foram apresentadas neste estudo, respectivamente, em termos de indícios fracos (-1), moderados (0) e fortes (1), dos aspectos revelados pelo teste.

Ressalta-se que as informações do referido manual são confidenciais e sua utilização é permitida somente para psicólogos com objetivos de diagnóstico e orientação de tratamento.

\section{Entrevista semi-estruturada}

A entrevista semi-estruturada utiliza questões previamente definidas, favorecendo o aprofundamento do conhecimento do paciente, a coleta dos dados e de outros aspectos não identificados através de testes, além de promover a uniformidade.

Neste estudo o roteiro da entrevista abordou inicialmente dados de caracterização dos sujeitos como idade, sexo, tempo de cirurgia e IMC. Em seguida, buscou-se investigar hábitos, atitudes e recursos utilizados para lidar com os estados emocionais relacionados ao comportamento alimentar antes e após a operação.

\section{Análise dos dados}

Os testes das Pirâmides Coloridas de Max Pfister, aplicados antes e após a operação, foram corrigidos e analisados através de avaliação quantitativa dos dados coletados.

Quanto aos dados da entrevista, foram estabelecidas categorias para uniformizar as informações obtidas quanto aos mecanismos referentes à compulsão antes e após a operação. As categorias de análise da entrevista foram hábito beliscador, preferência alimentar, motivo atribuído aos episódios compulsivos, resposta compensatória ao motivo.

Os dados dos testes e da entrevista foram submetidos à análise estatística para definir eventuais diferenças quanto aos indícios de compulsão nos períodos estudados. Para tanto utilizou-se teste de proporção $\mathrm{Z}$ para definir o perfil da população quanto aos indícios de compulsão antes e após a operação, adotando valor $P \leq 0,05$ de significância estatística. Foi utilizado para promover o estudo de concordância entre as mesmas variáveis avaliadas em momentos diferentes o método estatístico Kappa que apresentou valores estatisticamente significativos divididos em concordância baixa $(\mathrm{K} \leq 0,3)$, concordância moderada ( $\mathrm{K}$ entre $0,3-0,7)$ e concordância alta $(K \geq 0,7)$. Foi utilizado o teste Qui-Quadrado para avaliar os dados da entrevista com variáveis categóricas, adotando valor $P \leq 0,05$ de significância estatística.

\section{RESULTADOS}

A Tabela 1 apresenta detalhadamente os resultados do teste das Pirâmides Coloridas de Max Pfister. 
TABELA 1 - Dados referentes às formas das pirâmides realizadas antes e após a operação

\begin{tabular}{|c|c|c|c|c|c|c|c|c|c|c|c|}
\hline 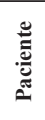 & & $\stackrel{\Xi}{\ddot{D}}$ & 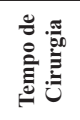 & 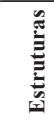 & & $\frac{Y}{2}$ & & $\sum$ & & ${ }^{+}$ & $\dot{A}$ \\
\hline & & & Meses & Pré & Pós & Pré & Pós & Pré & Pós & Pré & Pós \\
\hline 1 & 53 & $\mathrm{~F}$ & 50 & 0 & 0 & 1 & 0 & 1 & 1 & 0 & 0 \\
\hline 2 & 26 & $\mathrm{~F}$ & 39 & 1 & 0 & -1 & 0 & 1 & -1 & 0 & 0 \\
\hline 3 & 43 & $\mathrm{~F}$ & 40 & 0 & 0 & -1 & -1 & 0 & 0 & 1 & 1 \\
\hline 4 & 38 & $\mathrm{~F}$ & 45 & 0 & 0 & -1 & 1 & 0 & 1 & 0 & 0 \\
\hline 5 & 39 & $\mathrm{~F}$ & 29 & 0 & 0 & 1 & 0 & 0 & 1 & 0 & 0 \\
\hline 6 & 49 & $\mathrm{~F}$ & 47 & 0 & 0 & -1 & 1 & 1 & 0 & 0 & 0 \\
\hline 7 & 58 & M & 31 & 0 & 0 & 1 & 0 & -1 & 1 & 0 & 0 \\
\hline 8 & 41 & $\mathrm{~F}$ & 24 & 0 & 0 & -1 & 1 & 1 & 1 & 0 & 0 \\
\hline 9 & 55 & $\mathrm{~F}$ & 24 & 0 & 0 & 1 & 0 & 1 & 1 & 0 & 0 \\
\hline 10 & 22 & $\mathrm{~F}$ & 46 & 0 & 0 & -1 & -1 & 0 & -1 & 1 & 1 \\
\hline 11 & 47 & $\mathrm{~F}$ & 27 & & 0 & 0 & 0 & 0 & 1 & 0 & 0 \\
\hline 12 & 43 & M & 26 & 0 & 0 & -1 & 0 & -1 & 0 & 0 & 0 \\
\hline 13 & 58 & $\mathrm{~F}$ & 27 & 0 & 0 & 1 & 1 & -1 & -1 & 0 & 0 \\
\hline 14 & 44 & $\mathrm{~F}$ & 26 & 0 & 0 & 1 & 0 & 0 & -1 & 0 & 0 \\
\hline 15 & 43 & $\mathrm{~F}$ & 36 & 0 & 0 & 0 & 0 & 0 & 0 & 0 & 0 \\
\hline 16 & 35 & $\mathrm{~F}$ & 37 & 0 & 1 & -1 & -1 & 1 & 1 & 0 & 0 \\
\hline 17 & 31 & M & 24 & 0 & 0 & -1 & 1 & 1 & -1 & 0 & 0 \\
\hline 18 & 37 & $\mathrm{~F}$ & 50 & 0 & 0 & -1 & -1 & 1 & 1 & 0 & 0 \\
\hline 19 & 46 & $\mathrm{~F}$ & 24 & 1 & 1 & 1 & 0 & 1 & -1 & 0 & 0 \\
\hline 20 & 31 & $\mathrm{~F}$ & 24 & 0 & 1 & 1 & -1 & 1 & 0 & 0 & 0 \\
\hline 21 & 25 & $\mathrm{~F}$ & 34 & 0 & 0 & -1 & -1 & 0 & -1 & 0 & 0 \\
\hline 22 & 41 & $\mathrm{~F}$ & 28 & 0 & 0 & -1 & 0 & -1 & 1 & 0 & 1 \\
\hline 23 & 52 & $\mathrm{~F}$ & 37 & 0 & 0 & 0 & 0 & 0 & 0 & 0 & 1 \\
\hline 24 & 52 & $\mathrm{~F}$ & 39 & 1 & 0 & 1 & 1 & 1 & 1 & 0 & 0 \\
\hline 25 & 44 & $\mathrm{~F}$ & 50 & 0 & 0 & -1 & -1 & -1 & -1 & 0 & 0 \\
\hline 26 & 38 & $\mathrm{~F}$ & 50 & 0 & 0 & 0 & 0 & 1 & 0 & 0 & 0 \\
\hline 27 & 59 & $\mathrm{~F}$ & 36 & 0 & 0 & 0 & -1 & 0 & 0 & 0 & 0 \\
\hline 28 & 39 & $\mathrm{M}$ & 28 & 0 & 0 & -1 & -1 & 0 & 0 & 1 & 0 \\
\hline 29 & 34 & $\mathrm{~F}$ & 28 & 1 & 1 & -1 & 1 & -1 & 1 & 0 & 0 \\
\hline 30 & 37 & M & 48 & 0 & 0 & 0 & 1 & 0 & 1 & 0 & 0 \\
\hline 31 & 50 & $\mathrm{~F}$ & 26 & & 0 & -1 & -1 & 0 & 1 & 0 & 0 \\
\hline 32 & 41 & $\mathrm{~F}$ & 24 & 0 & 0 & 1 & 0 & -1 & -1 & 0 & 1 \\
\hline 33 & 52 & $\mathrm{~F}$ & 35 & 1 & 0 & 1 & 1 & -1 & -1 & 0 & 0 \\
\hline 34 & 65 & $\mathrm{~F}$ & 37 & 0 & 0 & -1 & 0 & 0 & 0 & 0 & 0 \\
\hline 35 & 32 & $\mathrm{~F}$ & 28 & 1 & 0 & 0 & 1 & -1 & 0 & 0 & 1 \\
\hline 36 & 45 & $\mathrm{~F}$ & 38 & 0 & 0 & 0 & 0 & 0 & 1 & 0 & 0 \\
\hline 37 & 26 & $\mathrm{~F}$ & 40 & 0 & 1 & 1 & 1 & -1 & 0 & 0 & 0 \\
\hline 38 & 45 & $\mathrm{~F}$ & 46 & 0 & 0 & 0 & 0 & 0 & 0 & 0 & 0 \\
\hline 39 & 51 & $\mathrm{~F}$ & 41 & 1 & 0 & 1 & 0 & -1 & 0 & 0 & 0 \\
\hline 40 & 44 & $\mathrm{~F}$ & 47 & 0 & 0 & -1 & 0 & 0 & 1 & 0 & 0 \\
\hline 41 & 24 & $\mathrm{~F}$ & 47 & 0 & 0 & -1 & 1 & -1 & 0 & 0 & 0 \\
\hline 42 & 38 & $\mathrm{~F}$ & 42 & 0 & 0 & 0 & 1 & 0 & 0 & 0 & 0 \\
\hline 43 & 45 & $\mathrm{~F}$ & 64 & 1 & 0 & -1 & -1 & 0 & 0 & 0 & 0 \\
\hline 44 & 47 & $\mathrm{~F}$ & 37 & 0 & 0 & 1 & 1 & 0 & 0 & 0 & 0 \\
\hline 45 & 40 & $\mathrm{~F}$ & 32 & 0 & 0 & 1 & 1 & 1 & 1 & 0 & 0 \\
\hline 46 & 38 & M & 69 & 0 & 0 & 1 & 0 & -1 & -1 & 0 & 0 \\
\hline 47 & 64 & $\mathrm{~F}$ & 52 & 0 & 0 & 0 & 0 & 0 & -1 & 0 & 0 \\
\hline 48 & 43 & $\mathrm{~F}$ & 26 & 0 & 0 & -1 & 1 & 1 & 0 & 0 & 0 \\
\hline 49 & 32 & $\mathrm{~F}$ & 24 & 0 & 0 & 1 & -1 & -1 & 0 & 0 & 0 \\
\hline 50 & 40 & M & 24 & 0 & 0 & 1 & -1 & -1 & 1 & 0 & 0 \\
\hline
\end{tabular}

Legenda 1 - sim; 0 - não

Legenda 1 - sim; 0 - não
Estruturas - indica capacidade emocional para lidar com situações conflitivas EZtruturas - indica capacidade emocional para lidar com sã

VM - cor vermelha avalia extroversão, irritabilidade, impulsividade, agressividade $\mathrm{MA}+\mathrm{PR}$ - dupla de cores avalia o risco de compulsão. Rigidez e inflexibilidade mental associado à falta de recursos para lidar com conflitos

A Tabela 2 detalha os resultados referentes aos hábitos e preferências alimentares, motivos atribuídos aos episódios compulsivos e respostas compensatórias, antes e após a operação.

\section{Análise Estatística}

O teste de proporção $\mathrm{Z}$ foi aplicado para obter o perfil populacional antes e após a operação e o de Kappa para análise individual, caso a caso. Os asterísticos representam significância estatística.
TABELA 2 - Resultados da entrevista semi-estuturada

\begin{tabular}{|c|c|c|c|c|c|c|c|c|c|c|c|}
\hline 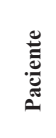 & 产 & 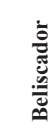 & 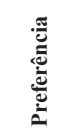 & 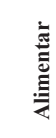 & 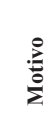 & & 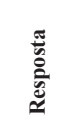 & 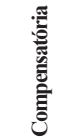 & & $\stackrel{+}{+\pi}$ & \pm \\
\hline & Pré & Pós & Pré & Pós & Pré & Pós & Pré & Pós & Pós & Pré & Pós \\
\hline 1 & 1 & & 1 & & 2 & 5 & 7 & 6 & 1 & 0 & 0 \\
\hline 2 & 1 & & 1 & 5 & 3 & 2 & 7 & 4 & -1 & 0 & 0 \\
\hline 3 & 1 & 0 & 3 & 2 & 3 & 3 & 7 & 2 & 0 & 1 & 1 \\
\hline 4 & 1 & 0 & 1 & 2 & 2 & 2 & 7 & 8 & 1 & 0 & 0 \\
\hline 5 & 1 & & 2 & 2 & 3 & 3 & 7 & 5 & 1 & 0 & 0 \\
\hline 6 & 1 & 1 & 1 & 2 & 2 & 2 & 7 & 2 & 0 & 0 & 0 \\
\hline 7 & 1 & & 4 & 2 & 3 & 3 & 7 & 1 & 1 & 0 & 0 \\
\hline 8 & 1 & 1 & 3 & 2 & 3 & 2 & 2 & 3 & 1 & 0 & 0 \\
\hline 9 & 1 & & 1 & 1 & 2 & 1 & 7 & 5 & 1 & 0 & 0 \\
\hline 10 & 1 & 1 & 1 & 3 & 3 & 2 & 1 & 4 & -1 & 1 & 1 \\
\hline 11 & 1 & 1 & 2 & & 2 & 2 & 7 & 3 & 1 & 0 & 0 \\
\hline 12 & 1 & & 1 & 2 & 2 & 2 & 7 & 3 & 0 & 0 & 0 \\
\hline 13 & 1 & 0 & 2 & 2 & 3 & 1 & 1 & 4 & -1 & 0 & 0 \\
\hline 14 & 1 & 0 & 3 & & 3 & 3 & 7 & 5 & -1 & 0 & 0 \\
\hline 15 & 1 & & 1 & 3 & 5 & 5 & 6 & 6 & 0 & 0 & 0 \\
\hline 16 & 1 & 0 & 2 & 2 & 3 & 2 & 7 & 5 & 1 & 0 & 0 \\
\hline 17 & 1 & 1 & 1 & 6 & 5 & 5 & 6 & 6 & -1 & 0 & 0 \\
\hline 18 & 1 & 1 & 3 & 1 & 4 & 5 & 1 & 6 & 1 & 0 & 0 \\
\hline 19 & 0 & 0 & 1 & 3 & 2 & 2 & 8 & 4 & -1 & 0 & 0 \\
\hline 20 & 1 & & 1 & 1 & 2 & 2 & 7 & 3 & 0 & 0 & 0 \\
\hline 21 & 1 & 0 & 4 & 1 & 5 & 5 & 6 & 6 & -1 & 0 & 0 \\
\hline 22 & 1 & & 2 & 1 & 3 & 3 & 7 & 2 & 1 & 0 & 1 \\
\hline 23 & 1 & 0 & 1 & & 5 & 5 & 6 & 6 & 0 & 0 & 1 \\
\hline 24 & 1 & 1 & 1 & 3 & 3 & 3 & 7 & 2 & 1 & 0 & 0 \\
\hline 25 & 1 & 1 & 1 & 2 & 5 & 5 & 6 & 6 & -1 & 0 & 0 \\
\hline 26 & 1 & 1 & 1 & 1 & 3 & 3 & 7 & 2 & 0 & 0 & 0 \\
\hline 27 & 1 & & 1 & 3 & 3 & 5 & 7 & 6 & 0 & 0 & 0 \\
\hline 28 & 1 & 0 & 2 & 1 & 3 & 1 & 7 & 5 & 0 & 1 & 0 \\
\hline 29 & 1 & 0 & 2 & 2 & 5 & 5 & 6 & 6 & 1 & 0 & 0 \\
\hline 30 & 0 & 1 & 4 & 2 & 2 & 2 & 7 & 3 & 1 & 0 & 0 \\
\hline 31 & 1 & 0 & 4 & & 5 & 5 & 6 & 6 & 1 & 0 & 0 \\
\hline 32 & 1 & 1 & 2 & 2 & 3 & 3 & 7 & 1 & -1 & 0 & 1 \\
\hline 33 & 1 & 1 & 1 & 2 & 5 & 5 & 6 & 6 & -1 & 0 & 0 \\
\hline 34 & 1 & 1 & 1 & 1 & 3 & 3 & 7 & 3 & 0 & 0 & 0 \\
\hline 35 & 1 & 1 & 4 & 2 & 3 & 3 & 7 & 2 & 0 & 0 & 1 \\
\hline 36 & 0 & 0 & 4 & 6 & 3 & 3 & 7 & 3 & 1 & 0 & 0 \\
\hline 37 & 1 & & 4 & 2 & 3 & 3 & 7 & 2 & 0 & 0 & 0 \\
\hline 38 & 1 & 1 & 3 & 3 & 1 & 1 & 7 & 5 & 0 & 0 & 0 \\
\hline 39 & 1 & 1 & 3 & 2 & 3 & 3 & 1 & 3 & 0 & 0 & 0 \\
\hline 40 & 1 & 0 & 2 & 2 & 3 & 3 & 7 & 3 & 1 & 0 & 0 \\
\hline 41 & 1 & 0 & 2 & 4 & 3 & 3 & 7 & 5 & 0 & 0 & 0 \\
\hline 42 & 1 & 1 & 3 & 2 & 1 & 1 & 1 & 1 & 0 & 0 & 0 \\
\hline 43 & 1 & 1 & 4 & 1 & 2 & 2 & 1 & 2 & 0 & 0 & 0 \\
\hline 44 & 1 & 1 & 1 & 2 & 3 & 3 & 7 & 1 & 0 & 0 & 0 \\
\hline 45 & 1 & 1 & 2 & 3 & 2 & 2 & 7 & 1 & 1 & 0 & 0 \\
\hline 46 & 1 & 0 & 1 & 6 & 2 & 2 & 7 & 3 & -1 & 0 & 0 \\
\hline 47 & 1 & 1 & 4 & 3 & 3 & 3 & 8 & 5 & -1 & 0 & 0 \\
\hline 48 & 1 & 1 & 2 & 3 & 5 & 5 & 6 & 6 & 0 & 0 & 0 \\
\hline 49 & 1 & & 2 & 3 & 3 & 3 & 7 & 3 & 0 & 0 & 0 \\
\hline 50 & 1 & 1 & 1 & 4 & 3 & 3 & 7 & 2 & 1 & 0 & 0 \\
\hline
\end{tabular}

Legenda - Hábito beliscador: 1 - sim; 0 - não

Preferência Alimentar: 1 - carboidratos; 2 -doces; 3 - petiscos; 4 - refeições (arroz, feijão, carnes); 5 - alimentos diet/light; 6 - cerveja

Motivos atribuídos: 1 - nervosismo; 2 - ansiedade/depressão; 3 - sentimento de impotência/ submissão; 4 - estímulo visual; 5 - não sabe referir

Respostas compensatórias: 1 - ingestão de doces; 2 -ingestão de petiscos; 3 - vontade de comer ; 4 - atividades sociais; 5 - expressão dos sentimentos; 6 - não sabe referir; 7 - comer indiscriminado; 8 - outros

\section{Análise do teste das Pirâmides Coloridas de Max Pfister}

Nesta análise, e no parâmetro que avaliou a estabilidade emocional e possibilidade de controle racional sobre os afetos e emoções, os resultados foram os seguintes: 1. teste Z, estabilidade e capacidade para lidar com conflitos em $16 \%$ dos pacientes antes da operação e em $10 \%$ após ela $(P=0,37) ; 2$. teste Kappa, aumento em $6,3 \%$ dos pacientes quanto à capacidade emocional para lidar com situações conflitivas e diminuição desta 
capacidade em 12,5\% após a operação, indicando funcionamento cognitivo e emocional alterado em relação ao período pré-operatório, com prejuízo na estabilidade emocional $(\mathrm{K}=0,206)$.

Quanto ao risco de compulsão os resultados foram: 1. teste $\mathrm{Z}$ de $6 \%$ antes da operação e $12 \%$ após $(P=0,29)$; 2. teste Kappa, $66,7 \%$ mantiveram-se em risco e $8,5 \%$ passaram a apresentar risco de compulsão após a operação $(\mathrm{K}=0,396)$.

Para a capacidade de controle e adaptação, os resultados foram: 1 . teste $\mathrm{Z}, 36 \%$ dos pacientes apresentavam fortes indícios de atitude de controle e rigidez antes da operação e $32 \%$ após $(P=0,67), 22 \%$ apresentavam moderada capacidade de controle e adaptação antes da operação e $42 \%$ após $(P=0,02), 42 \%$ apresentaram indícios de descontrole antes da operação e $26 \%$ após $(P=0,08) ; 2$. teste Kappa, em $30 \%$ dos pacientes houve aumento da atitude de controle e rigidez, sugerindo compulsividade, e em $26 \%$ houve prejuízo na capacidade de controle, sugerindo atitude descontrolada $(K=0,180)$.

Quanto aos indicadores de impulsividade, agressividade e irritabilidade, os dados revelaram que: 1 . teste $Z$, fortes indícios de atitudes agressivas e impulsivas em $28 \%$ antes da operação e em $36 \%$ após $(P=0,38)$, indícios moderados em $42 \%$ antes e $40 \%$ após $(P=0,83)$, fracos indícios destas características em 30\% antes e em $24 \%$ após a operação $(P=0,49) ; 2$. teste Kappa, a manifestação de agressividade, impulsividade e irritabilidade aumentou em $34 \%$ dos pacientes no período pós-operatório e em $22 \%$ diminuiu $(\mathrm{K}=0,150)$.

\section{Análise da entrevista}

Quanto ao comportamento beliscador, os dados mostraram: 1 . teste $\mathrm{Z}$ presente em $94 \%$ dos pacientes no pré-operatório e em $60 \%$ no pós $(P=0,31)$; teste Kappa, $33,3 \%$ adquiriram hábito beliscador após a operação e $63 \%$ permaneceram com comportamento beliscador $(\mathrm{K}=0,104)$.

Quanto à preferência alimentar, ela foi notada por alimentos calóricos como carboidratos (42\%), doces (26\%), petiscos (14\%) sem menção à bebidas alcoólicas no pré-operatório e no pós-operatório por alimentos de fácil ingestão como doces em $45 \%$ dos pacientes $(P=0,01)$, petiscos em $22 \%(P=0,31)$ e carboidratos em $20 \%(P=0,06)$, além de $7 \%$ referir preferência para cerveja $(P=0,06)$.

Quanto ao motivo atribuído aos episódios compulsivos, antes da operação $4 \%$ dos pacientes relataram estado de nervosismo como fator de desequilíbrio emocional que estimulava a ingestão excessiva de alimentos; $24 \%$ afirmaram distúrbios de humor como ansiedade e/ou depressão como fatores precipitantes; $52 \%$ identificaram sentimentos de impotência ou submissão diante de situações que não se sentiam capazes de resolver, compensados com o ato de alimentar. No pós-operatório, os mesmos motivos obtiveram respectivamente os índices $10 \%(P=0,23), 28 \%(P=0,64)$ e $38 \%(P=0,15)$.
Quanto às respostas compensatórias diante dos motivos atribuídos aos episódios compulsivos, os pacientes antes da operação recorriam a mecanismos que aliviassem o estado emocional desencadeado por tais fenômenos: ingestão de doces em $12 \%$ dos pacientes; ingestão de petiscos em $2 \%$ e comer indiscriminadamente tudo que estivesse ao alcance em $64 \%$ dos pacientes $(P \leq 0,001)$. Após o procedimento cirúrgico os pacientes não apresentavam capacidade de ingerir grandes quantidades, porém a alimentação permaneceu como recurso de alívio para situações conflitivas, seja através da ingestão de petiscos em $18 \%$ dos pacientes $(P=0,005)$ e de doces em $10 \%(P=0,74)$ ou do desejo de comer algo que tirasse o indivíduo do estado alterado em que se encontrasse em $22 \%(P \leq 0,001)$. Observou-se ainda que $16 \%$ dos pacientes passaram a expressar os sentimentos relativos ao conflito, solucionando a situação de outra forma que não através da comida $(P=0,002)$.

A associação entre a variável comer indiscriminadamente e desejo de comer como resposta compensatória ao evento estressor, respectivamente antes e após a operação, mostrou significância estatística $(P=0,005)$.

\section{DISCUSSÃO}

Os pacientes portadores de obesidade mórbida que apresentam compulsão diferem em vários aspectos dos não compulsivos e, a operação como recurso de tratamento para estes pacientes, influencia diferentemente na sua evolução.

Desta forma, os pacientes compulsivos podem representar uma subcategoria da população de obesos, apresentando níveis elevados de psicopatologia, como depressão e transtornos de personalidade, início precoce e maior gravidade da obesidade, e mais tempo dedicado a dietas, portanto um quadro clínico importante que deve receber atenção específical.

Os dados apresentados no presente estudo demonstraram características de personalidade antes e após a operação, identificando algumas mudanças entre os dois períodos. Observou-se características de compulsão em pacientes com estrutura emocional prejudicada, ansiedade, depressão, atitude controladora, dificuldade na elaboração das emoções, rigidez e busca da comida diante de situações conflitivas. Desta forma, a diminuição da ingestão alimentar poderia ter contribuído para alterações na estrutura emocional destes pacientes. Quanto às características dos obesos compulsivos, estudo comparando obesos e não-obesos identificando, na população obesa, características imaturas de personalidade reveladas por ansiedade, estrutura de personalidade frágil, depressão, inadequação na elaboração das relações, baixa auto-estima e dificuldade em tolerar culpa e frustração. Constatou ainda íntima relação entre a necessidade de comer e a dificuldade em lidar com os conflitos de maneira simbólica, considerando a obesidade necessária à manutenção do equilíbrio, cuja ruptura pode predispor transtornos psiquiátricos graves. 
A cirurgia bariátrica tem sido importante aliada na redução de peso e melhora das comorbidades de pacientes obesos, entretanto, existe a preocupação de que alterações do comportamento alimentar possam vir a trazer complicações pós-operatórias e comprometer o resultado da operação. Neste estudo verificou-se que a compulsão alimentar seria considerada fator de risco para essas complicações pós-operatórias, considerando que o paciente não ingere grandes quantidades de alimento, mas pode apresentar hábito beliscador. Este comportamento indica hábito alimentar que pode ser prejudicial à manutenção da perda de peso dependendo da qualidade dos alimentos ingeridos, portanto, considera-se o tratamento prévio do transtorno associado à obesidade um procedimento de grande importância para o sucesso da operação.

$\mathrm{O}$ ato de comer está relacionado a estímulos internos e externos, considerando fatores orgânicos, psíquicos e sociais. Neste sentido, a ingestão alimentar transcende a questão nutritiva, agregando motivações ocultas relacionadas às vivências conflituosas que independem da fome ${ }^{23}$. De modo semelhante os pacientes relataram desejo de comer, mesmo sem fome, diante das situações que os faziam comer indiscriminadamente antes da operação. Isto significa que, diante de situações que exijam organização emocional, eles possuem padrão de reação orientado pela comida, seja através da ingestão ou apenas da vontade. Desde muito cedo, estes pacientes aprendem a engolir medo, ressentimentos e a conter as emoções com comida, estabelecendo padrão de reação aos conflitos baseado na alimentação. Na população estudada observou-se que os pacientes submetidos à cirugia bariátrica desenvolvem novos padrões alimentares para conter as dificuldades emocionais, recorrendo a alimentos de fácil ingestão. Doces, torradas, bolachas e outros petiscos foram eleitos para exercer a função que a ingestão excessiva e descontrolada tinha antes da operação.

Os pacientes deste estudo apresentaram aumento nas atitudes de controle, talvez favorecido pela restrição imposta, porém o controle excessivo é considerado fator de risco à compulsão. A cirurgia bariátrica proporciona restrição para evitar que os pacientes alimentem-se em excesso, porém Bernardi et al. ${ }^{2}$ afirmam que as restrições parecem desencadear episódios compulsivos. Estudo similar apresentado por Lluch et al. ${ }^{15}$ reforça a idéia de que controle e restrição da ingestão alimentar pode, em algum momento, predispor o comportamento compulsivo. Alterações no funcionamento do sistema nervoso também são referidas indicando que a diminuição da ingestão alimentar reduz o tônus serotoninérgico central e predispõe o indivíduo à compulsão alimentar. Neste sentido, a análise realizada indica que a diminuição da ingestão alimentar e características como rigidez de pensamento, impulsividade e dificuldade na elaboração das emoções poderiam estar associadas na manifestação do comportamento compulsivo após cirurgia bariátrica.

O controle dos impulsos e da expressão das emoções apresenta-se alterado no pré e pós-operatório. Estudos demonstram que obesos controlam e encobrem a agressividade, além de apresentarem dificuldade para lidar com ansiedade e raiva. No presente estudo verificou-se que no período pós-operatório esta dificuldade parece permanecer, porém percebeu-se a expressão inadequada de raiva e agressividade, com índices elevados em relação ao pré-operatório. Estes sentimentos poderiam estar encobertos antes da operação pela ingestão excessiva de alimentos, de modo que a restrição alimentar favoreceu alterações de manifestação de raiva, agressividade e impulsividade.

Os pacientes estudados nesta pesquisa identificaram seus estados emocionais alterados e os mecanismos para lidar com estes. Entre os principais motivos atribuídos à compulsão encontrou-se ansiedade, depressão e sentimentos de submissão e impotência. Identificaram ainda a diferença ou não de reação a estes estados após a operação. Estudos com pacientes obesas indicam que comem excessivamente como mecanismo compensatório em situações de ansiedade, depressão, tristeza e raiva ${ }^{16,17}$. A projeção na comida abafa sentimentos de impotência ${ }^{8}$, observados nos pacientes estudados através do relato da sensação de incapacidade de solucionar problemas, sentimentos de baixa auto-estima e submissão. Diante da frustração que estes sentimentos geram, os pacientes sentem-se inferiorizados e recorrem à comida para compensar a sensação de incapacidade, como demonstrado através da análise da entrevista.

O padrão alimentar estudado apresentou preferências e alguns excessos no pós-operatório, como doces e petiscos. Já que a alimentação excessiva não é possível, passaram a comer mais, em intervalos curtos, alimentos de fácil ingestão e que possivelmente têm correlação com o estado emocional dos pacientes. Segundo Colles et al. ${ }^{12}$ obesos compulsivos frequentemente tornam-se "beliscadores" após a operação. Em estudo sobre as preferências alimentares de obesos compulsivos comparados com obesos não compulsivos, identificou-se consumo maior de doces e petiscos no primeiro grupo. Estudo com pacientes obesas indicou forte preferência por alimentos doces e ricos em gordura ${ }^{4}$.

Entre as preferências observadas nesta investigação através da entrevista, os pacientes citaram cerveja apenas para o período pós-operatório, uma vez que é ingerida com facilidade, além de inibir a fome. Em investigação com grande amostra populacional identificou-se a compulsão alimentar associada ao risco significativamente aumentado de dependência de álcool ${ }^{10,21}$.

Devido à alta prevalência de compulsão alimentar em obesos que procuram a cirurgia bariátrica, o diagnóstico e tratamento anterior poderá ter importante valor clínico, uma vez que o histórico prévio de compulsão alimentar está associado ao baixo índice de perda de peso após a operação de bypass ${ }^{3,22}$. Contudo não deve ser considerada fator impeditivo para a realização da operação, e sim, devidamente diagnosticada e acompanhada.

Neste estudo observou-se fatores de risco que indi- 
cam a possibilidade de que a população estudada apresente compulsão no pós-operatório; entretanto, estudos que utilizam escalas baseadas na definição do DSM-IV para episódios compulsivos referem ausência de compulsão alimentar após a operaçao ${ }^{21}$. Pesquisas devem chegar a consenso quanto à definição de compulsão alimentar após cirurgia bariátrica para que futuros estudos possam avaliar, a longo prazo, seu efeito no resultado da operação e vice-versa.

O prognóstico de compulsão alimentar em pacientes obesos graves que se submetem à cirurgia bariátrica é incerto. Não há consenso na literatura e dados precisos que garantam bom ou mau prognóstico adequadamente estudados e comprovados ${ }^{14,25}$. Os critérios de TCAP e episódios compulsivos precisam ser revisados e ampliados para que se obtenha mais precisão nas pesquisas e diagnósticos deste distúrbio ${ }^{11}$. Clareza na definição de comportamentos alimentares inadequados após opeação, métodos confiáveis para identificá-los e estratégias de intervenção pós-operatória são necessários para otimizar os resultados de perda de peso e promover o bem-estar psicológico dos pacientes.

\section{CONCLUSÃo}

Os pacientes portadores de obesidade mórbida submetidos à cirurgia bariátrica apresentam indícios de compulsão alimentar antes e após a operação. Ela está relacionada a aspectos psicológicos como dificuldade em organizar emoções, ansiedade, depressão e estrutura emocional prejudicada. Características de impulsividade, controle, rigidez e instabilidade emocional também contribuem para a manifestação de episódios compulsivos. Os hábitos e preferências alimentares modificam-se após a operação, de modo que os pacientes passam a consumir alimentos de fácil ingestão frente a situações que os faziam comer compulsivamente, não sendo observado trocas de compulsão. O comportamento alimentar inadequado persiste ou é agravado após a operação.

Machado CE, Zilberstins B, Cecconello I, Monteiro M. Binge eating before and after bariatric surgery. ABCD Arq Bras Cir Dig 2008;21(4):185-91

ABSTRACT - Background - Obesity is a complex, multifactorial disorder which comprises psychological, social and biological aspects and requires a multidisciplinary approach. It is considered to be a risk factor for binge eating disorder, in that the emotional dynamic of the obese individual would contribute to trigger the compulsive act. Aim - To analyze the signs of binge eating in patients who underwent bariatric surgery, before and two years after the surgery. Methods - A prospective study was conducted with 50 patients submitted to bariatric surgery by the Fobi-Capella technique. The instruments for psychological evaluation, applied before the surgery and two years after, were Max Pfister's Color Pyramid Test and a semi-structured clinical interview. The test was given before and after the surgery to identify evidence of compulsive behavior. The interview was conducted in the postoperative only. Results - The data showed stability and ability to cope with conflicts in $16 \%$ of the patients before and in $10 \%$ after the surgery. In terms of control and adaptation, $36 \%$ of the patients exhibited strong evidence of an attitude of control and rigidity before the surgery, and $32 \%$ postoperatively. Regarding the indicators of impulsivity, agressiveness and irritability, there were strong signs of aggressive and impulsive attitudes in $28 \%$ of the patients before the surgery and in $36 \%$ after. Grazing behavior was present in $94 \%$ of the patients preoperatively and in $60 \%$ postoperatively. Food preference was for high-calorie foods with no mention of alcoholic beverages in preoperative patients, and postoperatively, for foods which were easy to consume. Regarding the reason for the compulsive episodes, before the surgery, $4 \%$ of the patients reported uneasiness, $24 \%$ expressed mood disturbances and $52 \%$ identified feelings of impotence or submission. Postoperatively, the percentages were $10 \%, 28 \%$ and $38 \%$, respectively. As for compensatory responses, they consisted of the ingestion of sweet foods in $12 \%$, snack foods in $2 \%$ and indiscriminate eating in $64 \%$ of the patients. After the surgery, the percentages were $18 \%, 10 \%$ and $22 \%$. Conclusion - The patients exhibit signs of binge eating before and after the surgery. Such compulsion is related to psychological aspects. Eating habits and food preferences change after the surgery, as patients begin to eat foods which are easier to consume when facing situations that would make them eat compulsively; no addiction transfer was observed. The inadequate eating behavior persists or is aggravated after the surgery.

HEADINGS - Obesity. Obsession. Bariatric Surgery

\section{REFERÊNCIAS}

1. Azevedo AP, Santos CC, Fonseca DC. Transtorno da compulsão alimentar periódica. Rev Psiquiatr Clin. 2004;31(4):170-2.

2. Bernardi F, Cichelero C, Vitolo MR. Comportamento de Restrição Alimentar e Obesidade. Rev Nutr 2005;18(1)85-93.

3. Blaine B, Rodman J. Responses to weight loss treatment among obese individuals with and without BED: a matched-study meta-analysis. Eat Weight Disord. 2007 Jun;12(2):54-60.

4. Blundell JE, Gillett A. Control of food intake in the obese. Obes Res. 2001 Nov;9 Suppl 4:263S-270S.

5. Bocchieri-Ricciardi LE, Chen EY, Munoz D, Fischer S, Dymek-Valentine M, Alverdy JC, le Grange D. Pre-surgery binge eating status: effect on eating behavior and weight outcome after gastric bypass. Obes Surg. 2006 Sep;16(9):1198-204

6. Borges MB, Jorge MR, Morgan CM, Da Silveira DX, Custódio O. Bingeeating disorder in Brazilian women on a weight-loss program. Obes Res. 2002 Nov;10(11):1127-34

7. Borges MBF, Jorge MR. Evolução histórica do conceito de compulsão alimentar. Psiq Prat Med. 2000;33(4). [Acesso: 6/4/2008]. Disponível em: <http://www. unifesp.br/dpsiq/polbr/ppm/especial04.htm>
8. Bottino SMB, Junqueira C, Bairrão JFMH, Hanns LA., Rosa MD, Andrade LHSG. Transtornos da compulsão alimentar periódica e psicoterapia: é possível sistematizar a formulação psicodinâmica de caso? Rev Bras Psiquiatr. 2003;25(3):166-70.

9. Buddeberg-Fischer B, Bernet R, Sieber M, Schmid J, Buddeberg C. Epidemiology of eating behaviour and weight distribution in 14- to 19-year-old Swiss students. Acta Psychiatr Scand. 1996 Apr;93(4):296-304.

10. Bulik CM, Sullivan PF, Kendler KS. Medical and psychiatric morbidity in obese women with and without binge eating. Int J Eat Disord. 2002 Jul;32(1):72-8.

11. Colles SL, Dixon JB, O'Brien PE. Grazing and loss of control related to eating: two high-risk factors following bariatric surgery. Obesity (Silver Spring). 2008 Mar;16(3):615-22.

12. Colles SL, Dixon JB, O'Brien PE. Loss of control is central to psychological disturbance associated with binge eating disorder. Obesity (Silver Spring). 2008 Mar; 16(3):608-14.

13. Freitas S, Lopes CS, Coutinho W, Appolinario JC. Tradução e adaptação para o português da Escala de Compulsão Alimentar Periódica. Rev Bras Psiquiatr 2001;23(4):215-20

14. Latner JD, Clyne C. The diagnostic validity of the criteria for binge eating disorder. Int J Eat Disord. 2008 Jan;41(1):1-14. 
15. Lluch A, Herbeth B, Méjean L, Siest G. Dietary intakes, eating style and overweight in the Stanislas Family Study. Int J Obes Relat Metab Disord. 2000 Nov;24(11):1493-9.

16. Macht M. Characteristics of eating in anger, fear, sadness and joy. Appetite. 1999 Aug;33(1):129-39.

17. Macht M, Gerer J, Ellgring H. Emotions in overweight and normal-weight women immediately after eating foods differing in energy. Physiol Behav. 2003 Nov;80(2-3):367-74

18. Matos MIR, Aranha LS, Alessandra N. Compulsão alimentar periódica, ansiedade, depressão e imagem corporal em pacientes com obesidade grau III. Rev Bras Psiquiatr. 2002;24(4):165-9.

19. Matz PE, Foster GD, Faith MS, Wadden TA. Correlates of body image dissatisfaction among overweight women seeking weight loss. J Consult Clin Psychol. 2002 Aug:70(4):1040-4

20. Niego SH, Kofman MD, Weiss JJ, Geliebter A. Binge eating in the bariatric surgery population: a review of the literature. Int J Eat Disord. 2007 May;40(4):349-59.

21. Reichborn-Kjennerud T, Bulik CM, Sullivan PF, Tambs K, Harris JR. Psychiatric and medical symptoms in binge eating in the absence of compensatory behaviors. Obes Res. 2004 Sep;12(9):1445-54.
22. Sallet PC, Sallet JA, Dixon JB, Collis E, Pisani CE, Levy A, Bonaldi FL, Cordás TA. Eating behavior as a prognostic factor for weight loss after gastric bypass. Obes Surg. 2007 Apr;17(4):445-51.

23. Souto S, Ferro-Bucher JSN. Práticas indiscriminadas de dietas de emagrecimento e o desenvolvimento de transtornos alimentares. Rev Nutr. 2006;19(6):693704

24. Vitolo MR, Bortolini GA, Horta RL. Prevalência de compulsão alimentar entre universitárias de diferentes áreas de estudo. Rev Psiquiatr. 2006;28(1):20-6.

25. White MA, Masheb RM, Rothschild BS, Burke-Martindale CH, Grilo CM. The prognostic significance of regular binge eating in extremely obese gastric bypass patients: 12-month postoperative outcomes. J Clin Psychiatry. 2006 Dec;67(12):1928-35.

Fonte de financiamento: não há Conflito de interesse: não há Recebido para publicação: 25/06/2008 Aceito para publicação: 28/09/2008 